\title{
A Suggestion on the Estimation of Lattice-Decohesion of Metal due to Hydrogen
}

\author{
By Takehiko Watanabe*
}

\begin{abstract}
In this report, a conception was proposed to estimate the change in lattice-bond strength due to the presence of hydrogen in some metals.

A practical calculation was made in consideration of the Engel-Brewer theory which is an attempt to correlate the existing crystal structures with the electron configuration and the simple idea that the change in the number of unpaired electrons which take part in metallic bond causes the variation in interatomic bond strength when an electron added by hydrogen is introduced into d-band and s-band.

The tendency of the lattice-decohesion due to hydrogen for some metals estimated on the basis of the conception proposed in this study was in good agreement with that of their solubility of hydrogen, and so it was likely that this conception was of considerable reliability.
\end{abstract}

(Received May 13, 1976)

\section{Introduction}

It has been well known that steels and some kinds of metals are severely embrittled by hydrogen, and considerable efforts on this have been devoted to this problem by many workers. In consequence, the following theories concerning the mechanism of hydrogen-embrittlement have been proposed ${ }^{(1)(2)}$; that is, (A) hydrogen gas pressure theory, (B) hydrogen adsorption theory, (C) hydrogen-dislocation interaction theory, (D) lattice-decohesion theory, (E) hydride-precipitation theory.

In this report, the change in lattice-bond strength of metals by dissolved hydrogen has been estimated by a tentative based on the lattice-decohesion theory (D) ${ }^{(3)}$.

\section{A Basic Consideration of the Estimation of the Change in Lattice-Bond Strength}

At first, it is necessary to consider the state of electrons around a hydrogen atom. It is more likely that a hydrogen atom is slightly ionized and approaches the proton state ${ }^{(1)}$. That is to say, the $1 \mathrm{~s}$ electron of hydrogen is being transferred to the conduction band of the atom existing as a crystal or is taking part in the metallic bond.

* National Research Institute for Metals, Meguroku, Tokyo 153, Japan.
Therefore, it is expected that the number of electrons which take part in the metallic bond may be changed by the presence of dissolved hydrogen and then the interatomic bond strength should be changed.

The nature of the metallic bond which is needed here has not been known in detail as yet. It has been interpreted conceptually, however, that the metallic bond is a coulombic force between positive ions and conduction electrons in view of the ionic bond and/or covalent force between them, and d-, s-, and p-band electrons are taking part in the metallic bond.

In transition metals, the d-band electron plays an important role in metallic bond as shown in Fig. 1. It is well known that heats of dissociation of the metals on the 4th $\sim 6$ th periods have a maximum value at or around group $\mathrm{V}_{\mathrm{a}}$ or $\mathrm{VI}_{\mathrm{a}}$, and that the highest electron concentration results in the strongest bond strength, when the d-band is half filled.

For group $\mathrm{III}_{\mathrm{a}} \sim \mathrm{V}_{\mathrm{a}}$ transition metals of which d-bands are filled less than half, the number of unpaired electrons are increased, and when the excess electron from dissolved hydrogen is introduced into the d- and s-bands, the metallic bond should be strengthend. On the other hand, for group VI $_{a} \sim \mathrm{VIII}_{\mathrm{a}}$ transition metals, the number of unpaired electrons are decreased by an excess electron from hydrogen, and consequently the metallic bond should be weakened. This simple concept is a basis of

1977 Vol. 18 


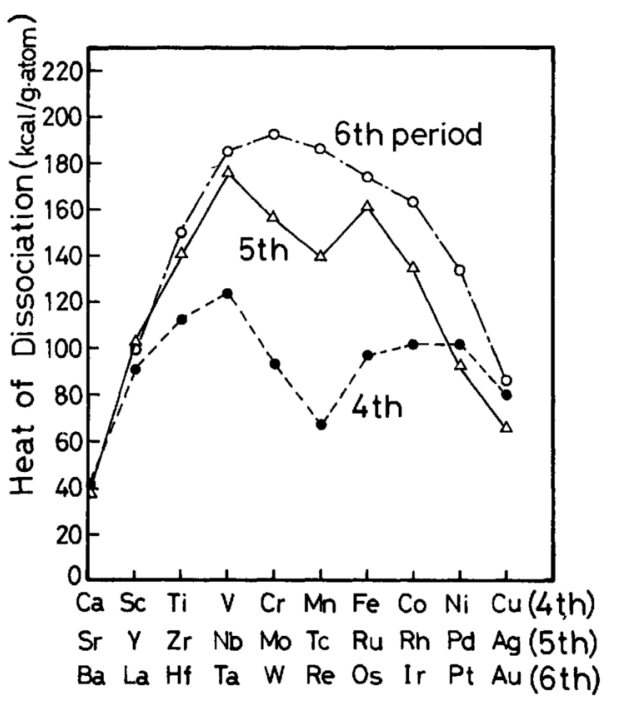

Fig. 1 Heats of dissociation for some transition metals of the 4 th, 5 th and 6 th periods.

the present work. The concept has also been proposed by Engel ${ }^{(4)}$ to explain the hydrogen solubility in transition metals, but he has not developed it furthermore.

When the change in lattice-bond strength due to hydrogen is discussed, it seems very useful to take into account the solubility data on hydrogen in transition metals, because the solubilities of hydrogen have a close relation with the change in the strength of the metallic bond. The lattice bond becomes more stable with increased interatomic bonding and more unstable with decreased unpaired electrons due to the existence of hydrogen.

\section{On the Solubility of Hydrogen in Transition Metals}

The following two mechanisms or models to explain the relation between the electronic structures and the solubility of hydrogen in transition metals have been proposed.

The electron concentration model ${ }^{(4)}$ is able to give a suitable explanation to the solubility of hydrogen in metals which have a group number lower than $\mathrm{V}_{\mathrm{a}}$ or have an electron concentration less than six, where the electron concentration represents the number of valence electron per atom, e/a. The smaller the e/a value, the larger becomes the solubility.

The density of states model ${ }^{(5)(6)}$, which is based on the change in the density of states at the Fermi surface of a solvent metal by dissolved hydrogen, may well account for the solubility of hydrogen in transition metals with the group number higher than $\mathrm{VI}_{\mathrm{a}}$. That is to say, the higher density of states at the Fermi surface, the larger becomes the solubility of hydrogen.

For the quantitative calculation of the change in lattice-bond strength by an excess electron, the Engel-Brewer theory is the most helpful.

\section{The Engel-Brewer Theory ${ }^{(4)(7)(8)}$}

The Engel-Brewer theory is an attempt to correlate the existing crystal structures with electron configurations of free atoms. For transition metals, this theory postulates that all
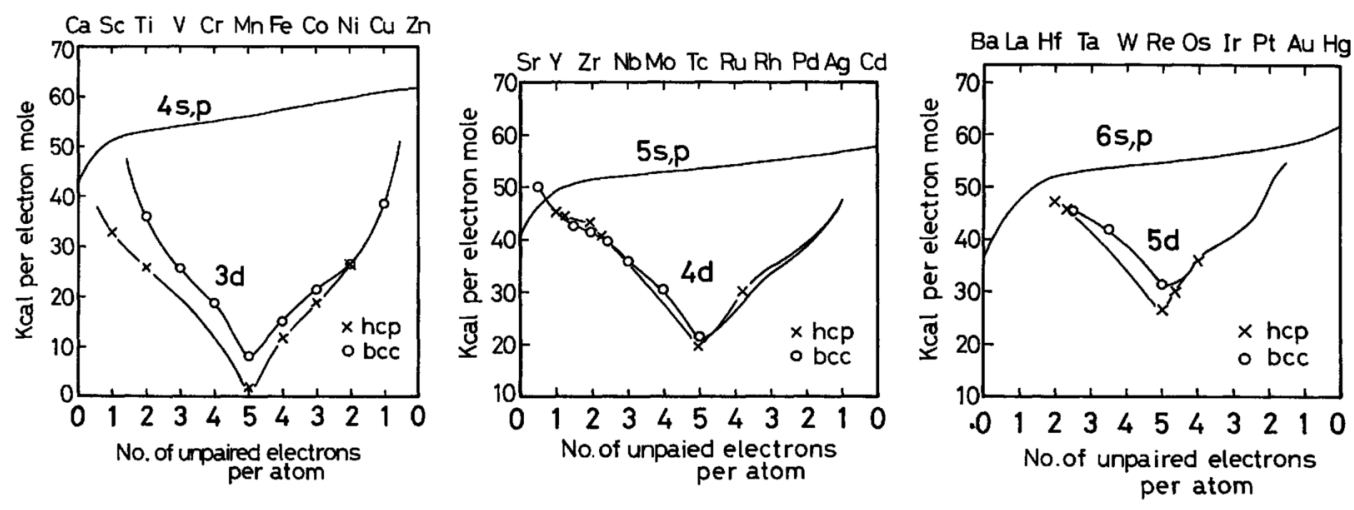

Fig. 2 Valence-state bonding enthalpy in kcal per mole per electron. Top curve: bonding enthalpy of $s$ or p electrons versus element. Bottom curve: bonding enthalpy of unpaired d electron (after Brewer ${ }^{(7)}$ ). 
the unpaired d-electrons take part in the metallic bond process, but play no part in controlling the type of crystal structure, which is determined solely by the number of $(s+p)$ electrons. That is, one, two and three $(s+p)$ electrons determine the $b c c, h c p$ and $f c c$ crystal structures of the metals, respectively. It is said that this theory can give a concise and satisfactory explanation of many properties of metals and alloys ${ }^{(8)}$.

An example of the application of the EngelBrewer theory to titanium will be given. The titanium atom in the ground state has the outer electronic configuration of $3 \mathrm{~d}^{2} \cdot 4 \mathrm{~s}^{2}\left(\rightarrow \mathrm{d}^{2} \mathrm{~s}^{2}\right)$. The titanium atom in a metallic state must have the outer electronic configuration of $3 \mathrm{~d}^{2} \cdot 4 \mathrm{~s} \cdot 4 \mathrm{p}$ $\left(d^{2} \mathrm{sp}\right)$ according to the Engel-Brewer theory. The process of condensation of titanium vapour to a metallic state may thus be regarded as involving two stages:

(1) The excitation process $3 d^{2} \cdot 4 s^{2} \rightarrow 3 d^{2}$. $4 s \cdot 4 p$, involving a promotion energy Ep. Ep is decided by a spectral experiment.

(2) The condensation process $3 \mathrm{~d}^{2} \cdot 4 \mathrm{~s} \cdot 4 \mathrm{p}$ gas $\rightarrow d^{2}$ sp crystal, involving a bonding enthalpy.

The atomization enthalpy is calculated with much ease by means of the drawings in Fig. 2 given by Brewer.

\section{Estimation of the Change in Interatomic Bond Strength due to Dissolved Hydrogen in Metals}

On the estimation of the change in latticebond strength due to hydrogen, which corresponds to the change in the atomization enthalpy, the distribution of an excess electron to the $\mathrm{d}$ - and s-bands of a metal should be considered ${ }^{\dagger}$.

For the estimation of the change in latticebond strength, it is more likely to do with a simillar idea to that on the solubility of hydrogen, which is described above.

For the transition metals with a group number lower than $V_{a}$, it is suitable that the

$\dagger$ It is postulated that the electron from hydrogen can be introduced into only $d$ - and s-bands because the p-band energy level is considerably higher than that of s- and d-bands. electron concentration model is adopted to determine the ratio of an electron added by hydrogen to be divided into d- and s-bands, and so the rate of variation of atomization enthalpy was calculated under the assumption that an electron is introduced into only the d-band so as to become more stable in the bonding state. Practical calculation will be illustrated by the case of titanium. As described above, the ground state of the outer electron configuration is $\mathrm{d}^{2} \mathrm{~s}^{2}$, and an excited state is $\mathrm{d}^{2} \mathrm{sp}$, involving a promotion energy of $46 \mathrm{kcal} /$ mol. From Fig. 2, it is seen that the bonding enthalpy of the $d^{2}$ sp valence state is $2 \times 26+$ $2 \times 53=158 \mathrm{kcal} / \mathrm{mol}$. The promotion energy of $46 \mathrm{kcal} / \mathrm{mol}$ yields an atomization enthalpy of $158-46=112 \mathrm{kcal} / \mathrm{mol}$. When an electron is added by hydrogen, the bonding enthalpy makes $(2+1) \times 20+2 \times 53=166 \mathrm{kcal} / \mathrm{mol}$, considering that an electron is introduced into only the d-band. In this case, the promotion energy of $46 \mathrm{kcal} / \mathrm{mol}$ yields an atomization enthalpy of $166-46=120 \mathrm{kcal} / \mathrm{mol}$. After all, an increase of the lattice-cohesion occurs, and the rate of increase is $(120-112) \times 100 / 112=$ $7.1(\%)$.

For the transition metals with a group number higher than $\mathrm{VI}_{\mathbf{a}}$, the change in atomization enthalpy was calculated in consideration of the density of states model. The density of states at the Fermi surface of a metal, $N(E)$, is connected with the coefficient of electronic specific heat, $\gamma$, by the equation, $\gamma=2 / 3 \pi^{2} k^{2}$ $N(E) / \rho^{(9)}$, where $k$ is the Boltzmann constant and $\rho$ is the density. There is a linear correlation between the density of states and the coefficient of electronic specific heat. The densities of states at the Fermi surface for some metals ${ }^{(10)}$ calculated from the experimental data on the electronic specific heat are shown in Fig. 3.

Figures 4 and 5 indicate the density of states curves of $3 d$-band and $4 \mathrm{~s}$-band electrons for nickel $^{(11)}$ and copper ${ }^{(12)}$, respectively. It is seen that $\mathrm{d}$-band electrons are distributed in a narrow region with high density, and that the density of states of s-band electrons is considerably low because of their scattering over a broad energy region.

For the metals, such as nickel, shown in Fig. 4, of which the density of states of the 3d- 
band at the Fermi surface is high, the electron added from hydrogen is apt to be introduced into the d-band. This is ascribed to the reason

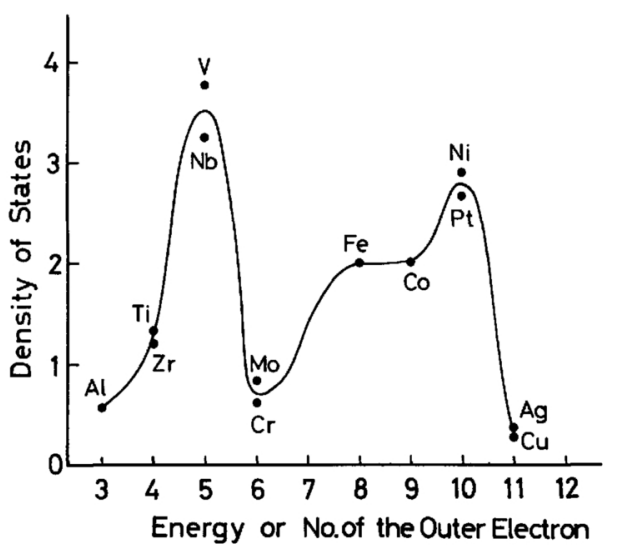

Fig. 3 Densities of states at the Fermi surface of some metals (after Burch(10)).

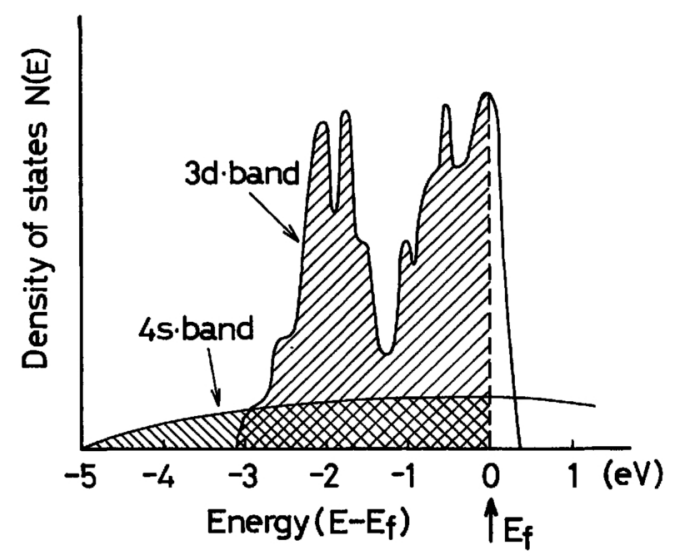

Fig. 4 Density of states curves for nickel (after Koster $\left.^{(11)}\right)$.

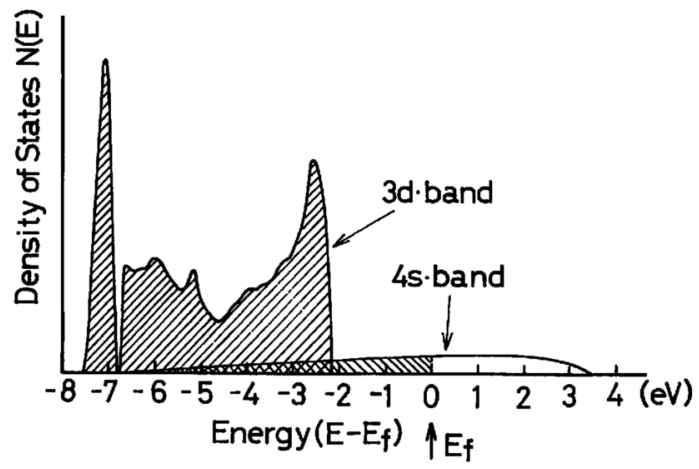

Fig. 5 Density of states curves for copper (after Seitz $\left.{ }^{(12)}\right)$.
Table 1 Density of states, electronic specific heat and $\{N(E)-0.3\} \times 34$ for some metals.

\begin{tabular}{lccc}
\hline \hline & $\begin{array}{c}\text { Density of } \\
\text { states } N(E), \\
\text { eV }^{-1} \cdot \text { atm }^{-1}\end{array}$ & $\begin{array}{c}\text { Electronic } \\
\text { specific } \\
\text { heat } \\
\times 10^{4} \mathrm{cal}^{\prime} \\
\mathrm{mol}^{\prime} / \mathrm{deg}^{2}\end{array}$ & $\{N(E)-0.3\} \times 34$ \\
\hline $\mathrm{Ni}$ & 2.90 & 17.40 & 88.40 \\
$\mathrm{Fe}$ & 2.00 & 12.00 & 57.80 \\
$\mathrm{Al}$ & 0.58 & 3.48 & 9.52 \\
$\mathrm{Cu}$ & 0.29 & 1.76 & 0.00 \\
$\mathrm{Cr}$ & 0.63 & 3.80 & 11.22 \\
$\mathrm{Co}$ & 2.00 & 12.00 & 57.80 \\
$\mathrm{Pt}$ & 2.67 & 16.00 & 80.58 \\
\hline \hline
\end{tabular}

that the change in the Fermi energy is relatively small, even if electrons are introduced into the d-band. For this reason, the density of states of the d-band was used as an index of the tendency for electrons added from hydrogen to be introduced into the 3d-band.

From the density of states curves of copper shown in Fig. 5, it is seen that d-band is fully filled and the s-band is half filled with electrons. As a result, the density of states of copper at the Fermi surface consists of only s-band electrons, and consequently its value is very small, $0.29 \mathrm{eV}^{-1} \cdot \mathrm{atm}^{-1}$, as indicated in Table 1 .

Therefore, for the metals of which the d-band is not fully filled, it was postulated that the proportion of the density of states occupied by s-band electrons within the total of the density of states at the Fermi surface was about $0.3 \mathrm{eV}^{-1} \cdot \mathrm{atm}^{-1}$, and that the value of $\{N(E)-$ $0.3\}$ was regarded as the density of states of dband electrons. So, in this work, the value of $\{N(E)-0.3\}$ was used as an index for an added electron from hydrogen to be distributed to dand s-bands.

In practice, assuming that the electron was introduced into the d-band in the proportion of $\{N(E)-0.3\} \times 34(\%)$ multiplied by an optimum coefficient of 34 , the variation of the atomization enthalpy due to hydrogen was calculated.

The coefficient of 34 was chosen for the following reason. In several metals with a group number higher than $\mathrm{VI}_{\mathrm{a}}$ except for $\mathrm{Mn}$ and $\mathrm{Pd}$ having abnormal properties, nickel has the highest density of states at the Fermi 
surface of $2.9 \mathrm{eV}^{-1} \cdot \mathrm{atm}^{-1}$ and copper has the lowest value of $0.29 \mathrm{eV}^{-1} \cdot \mathrm{atm}^{-1}$. As the ratio of the density of states of $\mathrm{Cu}$ to that of $\mathrm{Ni}$ is just $1: 10$, it is postulated that $90 \%$ of an added electron by hydrogen will be introduced into the d-band and $10 \%$ of the electron will be introduced into the s-band. For this reason, the coefficient of 34 was determined for $90 \%$ electron added by hydrogen to be introduced into the d-band. In the case of $\mathrm{Ni},\{N(E)-0.3\}$ $\times 34=88.4 \simeq 90(\%)$.

The electronic specific heats, the densities of states and the values of $\{N(E)-0.3\} \times 34(\%)$ are shown in Table 1.

An example of the calculation is illustrated by the case of nickel. The ground state of the nickel atom has the outer electronic configuration of $\mathrm{d}^{8} \mathrm{~s}^{2}$ and the excited state of $f c c \mathrm{Ni}$ has that of $\mathrm{d}^{7} \mathrm{sp}^{2}$, involving a promotion energy of $131 \mathrm{kcal} / \mathrm{mol}$. The bonding enthalpy is $3 \times 19+$ $3 \times 59=234 \mathrm{kcal} / \mathrm{mol}$. The promotion energy of $131 \mathrm{kcal} / \mathrm{mol}$ yields an atomization enthalpy of $234-131=103 \mathrm{kcal} / \mathrm{mol}$.

When an electron is added from hydrogen to nickel, the bonding enthalpy becomes (3$0.884) \times 26+(3-(1-0.884)) \times 59=225.17 \mathrm{kcal} /$ mol, considering that $88.4 \%$ of one electron will be introduced into the d-band for the reason mentioned above. In the case of nickel with dissolved hydrogen, the promotion energy

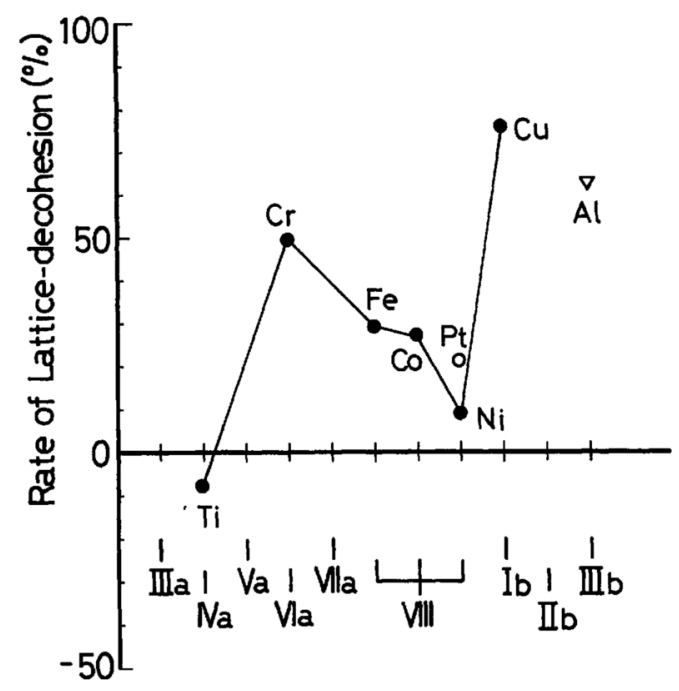

Fig. 6 The rates of lattice-decohesion due to hydrogen for some metals.

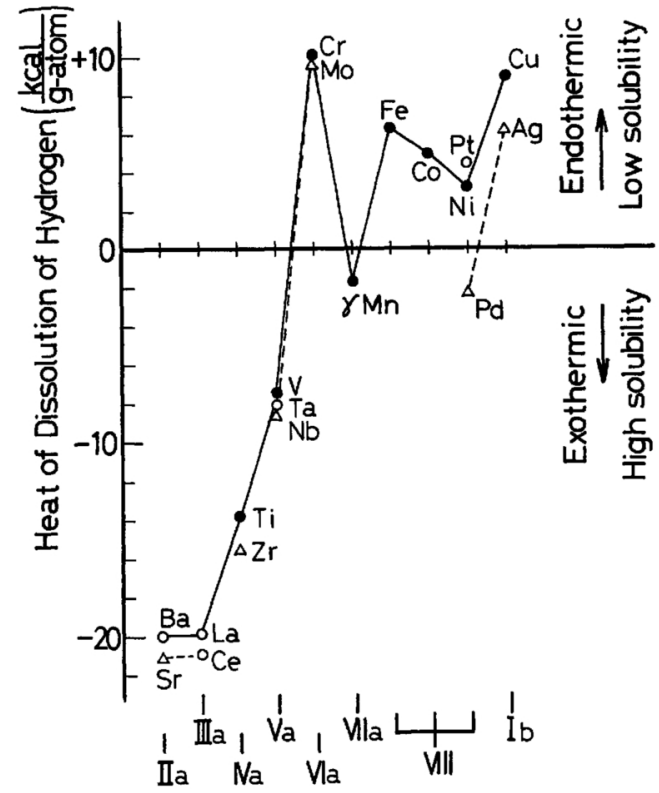

Fig. 7 Variation of heat of dissolution of hydrogen in transition metals (after Ebisuzaki and O'Keeffe ${ }^{(5)}$ ).

yields an atomization enthalpy which was calculated to be $225.17-131=94.17 \mathrm{kcal} / \mathrm{mol}$ by using the promotion energy of pure nickel. So the lattice-cohesion decreases, and the rate of the decrease is $(103-94.17) \times 100 / 103=8.57(\%)$.

The change in atomization enthalpy for some metals, the rate of lattice-decohesion, are calculated in this manner and the results are shown in Fig. 6.

The estimation of the change in lattice-bond strength seems to have a considerable reliability in spite of the crude assumptions in that it can be seen the clear interrelation between the tendency of the variation of lattice-decohesion shown in Fig. 7 and heats of dissolution of hydrogen or the solubility of hydrogen in some transition metals shown in Fig. 6.

\section{Conclusion}

The present work suggests that it is possible to estimate the change in the lattice-bond strength due to hydrogen in transition metals mainly, in consideration of the behaviour of d-band electrons and by adopting the EngelBrewer theory. 


\section{Acknowledgments}

The author wishes to express his gratitude to Dr. Keikichi Nakamura, National Research Institute for Metals, for many valuable discussions.

\section{REFERENCES}

(1) E. Fujita: Mechanism of Hydrogen-induced delayed Cracking, Ed. by Delayed Cracking Committee, the joint group for basic Research of Iron and Steel, JISI (1975), 10, 5 .

(2) M. Nagumo: Metal and Hydrogen, Ed. by Japan Institute of Metals, (1974), p. 106.

(3) For example, A. R. Troiano: Trans. ASM, 52 (1960), 54.

(4) N. N. Engel and J. E. Johnston: Preprint,
Congrés International, Paris, 29 mai-2 juin (1972), 60.

(5) Y. Ebisuzaki, M. O'Keeffe: Progress in Solid State Chemistry, Pergamon Press, New York, 4 (1967), 187.

(6) T. Eguchi, S. Morozumi: J. Japan Inst. Metals, 38 (1974), 1025.

(7) L. Brewer: Phase Stability in Metals and Alloys, McGraw-Hill, New York, (1967), p. 39.

(8) K. Nakamura: Kagakuno Ryoiki, 29 (1975), 68.

(9) K. Adachi et al.: Kinzoku no Denshi Ron I, AGNE, (1969), p. 43.

(10) R. Burch: Trans. Farady Society, 66 (1970), 736.

(11) G. F. Koster: The Physical Rew., 98 (1955), 901.

(12) F. Seitz: The Physics of Metals, McGraw-Hill, New York and London, (1943), p. 271. 